\title{
Revitalisierung der Gewerkschaften? Die Grundlagen amerikanischer Organisierungserfolge und ihre Übertragbarkeit auf deutsche Verhältnisse
}

\author{
Britta Rehder
}

Zusammenfassung: Seit den 1980er Jahren haben einige US-amerikanische Gewerkschaften durch neue Strategien und politische Bündnisse unerwartete Mitgliederzuwächse erzielt. Die deutsche Dienstleistungsgewerkschaft ver.di versucht, aus den amerikanischen Erfahrungen zu lernen. Der Artikel untersucht zentrale Voraussetzungen, auf denen die amerikanischen Organisierungserfolge beruhen, und er prüft, ob eine Übertragbarkeit auf deutsche Verhältnisse möglich ist. Zudem diskutiert er die normativen Implikationen des Lernens am amerikanischen Gewerkschaftsmodell.

Schlüsselwörter: Gewerkschaften · Korporatismus · Lernende Organisation · Mitgliederrekrutierung $\cdot$ Mobilisierung $\cdot$ Kampagnenpolitik

\section{Union Revitalization in Germany: Lessons to be Learnt from the American Experience}

\begin{abstract}
Since the 1980s some U.S.-American unions have been rather successful in recruiting new members through strategic campaigns. German unions have tried to emulate these strategies. This article analyses the institutional and normative foundations of the „American way of organizing". It tries to answer the questions whether it is feasible and desirable to import the strategies to the German context.
\end{abstract}

Keywords: Unions · Corporatism · Learning Organization · Organizing · Mobilization · Campaigning

Résumé: Depuis les années 1980, plusieurs syndicats américains ont enregistré un afflux d'adhérents inattendu grâce à de nouvelles stratégies et alliances politiques. Le syndicat allemand du secteur des services, ver.di, tente de tirer les leçons des expériences américaines. Cet article étudie les conditions essentielles sur lesquelles repose le succès des organisations américaines et s'interroge sur la possibilité de les transposer dans le contexte allemand. Nous discutons en outre les implications normatives de l'apprentissage à partir du modèle syndical américain.

Mots-clés: syndicats $\cdot$ corporatisme $\cdot$ organisation intelligente $\cdot$ recrutement d'adhérents mobilisation $\cdot$ politique de campagne

Britta Rehder $(\bowtie)$

Wissenschaftliche Mitarbeiterin am Max-Planck-Institut für Gesellschaftsforschung, Paulstraße 3, 50676 Köln, Deutschland

E-Mail: rehder@mpifg.de 


\section{Einleitung}

Gewerkschaften sind für Sozialwissenschaftler wieder interessant geworden. Jahrelang hatte die Verbändeforschung vor allem ihren Niedergang dokumentiert. Gewerkschaften galten als „Dinosaurier der Dienstleistungsgesellschaft“ (Ebbinghaus 2002). Der wirtschaftliche Strukturwandel, die Pluralisierung von Arbeitnehmeridentitäten, die steigende Massenarbeitslosigkeit und viele Gründe mehr wurden herangezogen, um zu erklären, dass die Gewerkschaften heute kaum mehr umfassende Interessenvertretungsmonopole der Arbeitnehmer sind (z.B. Regini 1992; Martin/Ross 1999). Allein in Deutschland haben sie seit 1992 mehr als vier Millionen Mitglieder verloren. Nur etwa jeder fünfte aktiv Beschäftigte ist heute noch gewerkschaftlich organisiert (Schnabel/Wagner 2007: 121). Gleichzeitig nahm auch der Einfluss der Gewerkschaften ab. Die Prägekraft von Tarifverträgen ist stark rückläufig (Ellguth/Kohaut 2007). Und in politischen Entscheidungsprozessen wurde die Rolle der Gewerkschaften nicht nur in Deutschland auf die einer Interessengruppe unter vielen reduziert.

Doch seit einigen Jahren finden sich vermehrt Hinweise in der Literatur, dass die Arbeitnehmerverbände zu neuem Leben erwachen (z.B. Bronfenbrenner et al. 1998; Frege/Kelly 2003; Hälker/Vellay 2006). Die Entwicklung hat dort begonnen, wo man es am wenigsten erwartet hätte: ausgerechnet in den USA, wo die Gewerkschaften mit einem Organisationsgrad von rund $9 \%$ nahezu totgesagt worden waren (Milkman/Voss 2004: 1); und ausgerechnet im Dienstleistungssektor, in dem doch für gewerkschaftliche „Dinosaurier“ kein Platz mehr zu sein schien. Die Arbeitnehmerorganisationen versuchen, sich mit neuen Strategien zu regenerieren. Dies bedeutet vor allem, Mitgliederzuwächse zu erzielen, um als Organisation zu überleben und politische Forderungen kraft eigener Stärke durchzusetzen.

Das prominenteste Fallbeispiel ist die amerikanische Dienstleistungsgewerkschaft SEIU (Service Employees International Union), der es gelang, lateinamerikanische Immigranten zu organisieren. Mit dem Aufstieg des SEIU-Präsidenten John Sweeney zur Leitfigur der gewerkschaftlichen Dachorganisation AFL-CIO rückte die Mobilisierungsstrategie an die Spitze der gewerkschaftlichen Agenda. Erfolge zeigt sie vor allem im Gesundheits- und Bildungswesen sowie im Sicherheitsdienst, im Hotel-, Reinigungsund Restaurantgewerbe (Bronfenbrenner/Hickey 2004: 1 ff.). Die Revitalisierung der Gewerkschaften scheint weniger darin zu bestehen, dass die Erfolge quantitativ sehr beeindruckend sind. Das Organisationsniveau wurde in den USA bestenfalls stabilisiert. Das revitalisierende Element besteht (bisher) weniger in den Effekten als in den Strategien: Die Gewerkschaften erstarren nicht länger in Routine, sondern sie versuchen durch strategisches Handeln, ihr Überleben zu sichern.

Die Neuorientierung der Gewerkschaften wird in der Literatur unterschiedlich bezeichnet, wobei die begrifflichen Differenzen auch eine inhaltliche Bedeutung haben. Einige Autoren beschreiben die Revitalisierung als einen Prozess, in dem die Arbeitnehmerverbände ihre Wurzeln als soziale Bewegung wiederentdecken und ähnlich wie eine solche agieren (,Social Movement Unionism“, vgl. z. B. Turner/Hurd 2001: 9 f.). Andere Autoren betonen hingegen die Bedeutung neuartiger Verbandsstrategien in Richtung einer offensiven Mitgliedergewinnung (,organizing“ oder sogar „strategic organizing“, vgl. z. B. die Beiträge in Bremme/Fürniß/Meinecke 2007a). Unabhängig von der Be- 
zeichnung spielen drei Komponenten eine zentrale Rolle: die externe Mobilisierung zur Rekrutierung neuer Mitglieder, die interne Mobilisierung der Basis zur Unterstützung dieses Ziels sowie die Bildung neuer Koalitionen mit politischen Akteuren. Auch taktisch fand eine Erneuerung statt. US-Gewerkschaften treten heute weniger durch Streiks und Demonstrationen hervor, sondern sie organisieren strategische Kampagnen gegen Unternehmen, wie sie vor allem von Nichtregierungsorganisationen bekannt sind, um die Öffentlichkeit für Arbeitnehmerinteressen zu sensibilisieren.

Die Revitalisierungsbemühungen haben in anderen angelsächsischen Ländern Nachahmer gefunden (Oxenbridge 1997; Heery et al. 2000). Und auch bei kontinentaleuropäischen Gewerkschaften erlangt die neue Strategie Aufmerksamkeit (vgl. die Beiträge in Frege/Kelly 2003). Das markanteste deutsche Beispiel bietet die Dienstleistungsgewerkschaft ver.di, die in Kooperation mit anderen Organisationen verschiedene Kampagnen durchführte, u.a. gegen den Schwarz-Konzern und seine Supermarktkette „Lidl““.

Dass US-amerikanische Strategien bei den deutschen Gewerkschaften auf Resonanz stoßen, ist nicht nur praktisch-politisch, sondern auch theoretisch interessant. Denn wir erleben hier ,den organisationssoziologisch eigentlich unwahrscheinlichen Fall, dass bürokratische Apparate den institutionell scheinbar vorgegebenen Pfad organisationaler Entwicklung verlassen und in vergleichsweise kurzer Zeit zu einer grundlegenden Erneuerung ihrer Praxisformen gelangen“ (Dörre 2006: 15). Drei Aspekte sind dabei von besonderem Interesse:

1. Vor dem Hintergrund der Literatur über die Spielarten des Kapitalismus (vgl. die Beiträge in Hall/Soskice 2001a) ist es nicht (mehr) überraschend, wenn sich die deutschen Gewerkschaften amerikanisieren. Vielmehr passt diese Entwicklung in das Bild, das die Forschung von der Liberalisierung des „Rheinischen Kapitalismus“ zeichnet. Wenn in der Literatur auch keine Einigkeit darüber besteht, dass die Liberalisierung auch Amerikanisierung bedeutet, so stimmen die Analysen doch dahingehend überein, dass der „rheinische Kapitalismus“ einem grundlegenden Wandel unterliegt. Die Entwicklung der deutschen Gewerkschaften veranschaulicht, wie politische Akteure unter der Bedingung der schwindenden Prägekraft institutioneller Arrangements neue Handlungsorientierungen entwickeln. Dass die Gewerkschaften dabei auf US-Importe zurückgreifen, eröffnet die Möglichkeit, die Funktionsweise „fremder“ Strategien in „heimischen“ Kontexten zu studieren.

2. Bei der Neupositionierung der deutschen Gewerkschaften handelt es sich um den relativ seltenen Fall des expliziten Organisationslernens. Dabei wird ein durchaus anspruchsvolles Lernprogramm avisiert, das dem Konzept des „double-loop learning“ entspricht (Argyris/Schön 1999: 36). Bandelow beschreibt das Doppelschleifen-Lernen als ein Programm, in dem ,ein Akteur bestimmte Ziele seines Handelns verändert, um die Erreichung von noch grundlegenderen Zielen zu ermöglichen“ (2003: 302). Übertragen auf das Beispiel von ver.di bedeutet dies, die Gewerkschaft definiert ihre Ziele dahingehend neu, dass sie sich nicht mehr nur auf das gewerkschaftliche Kerngeschäft (Tarifverhandlungen) konzentriert, und vor allem nicht dort, wo ein gewerkschaftlicher Zugang zu den Betrieben bereits existiert. Vielmehr wird darauf abgestellt, sich mit neuen Methoden in bisher weitgehend gewerkschaftsfreien Wirtschaftsbereichen zu etablieren. Diese (zumindest partielle) Neudefinition der Ziele dient dazu, das grundlegendere Ziel der Organisationssicherung zu verwirklichen. Explizit ist das Organisationslernen 
insofern, als sich hier nicht nur eine beiläufige Diffusion gewerkschaftlicher Strategien aus anderen Ländern vollzieht. Ver.di will lernen: Man hat sich explizit darum bemüht, Expertise aus den USA zu gewinnen. Vor diesem Hintergrund ist die grenzüberschreitende Diffusion kein Zufall, sondern Bestandteil einer gewerkschaftlichen Transnationalisierungsstrategie (Greven 2006: $257 \mathrm{f}$.).

3. Die politische Entwicklung ist auch deshalb theoretisch interessant, weil sie der Forschung über die Determinanten gewerkschaftlicher Organisationsmacht nicht entspricht. In vielen Arbeiten wurde das Augenmerk auf die zyklischen, strukturellen und institutionellen Faktoren gewerkschaftlicher Mobilisierung gelegt (z.B. Ebbinghaus/ Visser 1999: 138 ff.). Schon Mancur Olson hatte in seinem berühmten Werk über die Logik des kollektiven Handelns argumentiert, Gewerkschaften könnten aus eigener Kraft kaum überleben (1968: 65 ff.). Selektive Anreize, vor allem aber institutionelle Bedingungen, die faktisch als Organisationshilfen wirken, bilden nach Olson unverzichtbare Voraussetzungen zur Überwindung des Kollektivgutproblems. Die aktuellen Revitalisierungsstrategien arbeiten hingegen mit der Prämisse, dass die Gewerkschaften (gegenwärtig) mit keinem der Olson'schen Instrumente Nutzen generieren können. Sie profitieren gerade nicht von institutionell generierter Absicherung. Und Serviceangebote, wie z.B. Versicherungen, Beratung oder Freizeitangebote, müssen tendenziell reduziert werden, wenn ein größerer Teil der finanziellen Ressourcen in die Kampagnenpolitik umgelenkt wird. Der Ansatz betont, dass Strategien zum Aufbau genuiner Organisationsmacht die Voraussetzung für institutionelle Anerkennung sind.

Aus den genannten Gründen widmet sich dieser Aufsatz der Frage, ob und wie das US-amerikanische Konzept der Revitalisierung in Deutschland funktionieren kann. Dabei geht es nicht um eine Prognose der Erfolgsaussichten, die bei Reformexperimenten in dieser Größenordnung immer unsicher sind. Vielmehr sollen die Voraussetzungen und Bedingungen erfolgreicher US-amerikanischer Kampagnen herausgearbeitet werden. Einige Befürworter der amerikanischen Strategien führen die deutsche Kritik am Konzept auf einen vermeintlichen Anti-Amerikanismus zurück (Stövesand 2007: 83; Bremme 2007: 215). Dieser spielt sicherlich eine Rolle. Es ist nicht selbstverständlich, dass die deutschen Gewerkschaften einerseits den Kapitalismus amerikanischer Provenienz so vehement bekämpfen, sich dann aber andererseits ausgerechnet an amerikanischen Gewerkschaftsstrategien orientieren. Jenseits der kulturellen Vorbehalte kann jedoch kein Zweifel daran bestehen, dass die Funktionsweise der US-Revitalisierungsversuche auf spezifischen institutionellen, strukturellen und vor allem normativen Grundlagen basiert, über die es sich lohnt, länger nachzudenken. In diesem Aufsatz wird untersucht, ob diese Grundlagen auch in Deutschland vorliegen bzw. hergestellt werden können.

Der Artikel ist folgendermaßen aufgebaut. Die deutschen und amerikanischen Arbeitsbeziehungen zu vergleichen, bedeutet, sehr unterschiedliche Systeme einander gegenüberzustellen. Darum werden in Abschnitt 2 die hier relevanten und grundlegenden institutionellen Unterschiede zwischen beiden Systemen skizziert. Abschnitt 3 diskutiert die zentralen Elemente gewerkschaftlicher Revitalisierungsstrategien, auch wenn das Konzept weit davon entfernt ist, ein theoretisches Modell abzubilden (Frege 2000: 266 ff.). Wie bereits erwähnt wurde, ist selbst der Name umstritten. Abschnitt 4 stellt zwei Fallbeispiele vor: die „Justice for Janitors“-Kampagne der Gewerkschaft 
SEIU in Südkalifornien sowie die „Lidl“-Kampagne der Dienstleistungsgewerkschaft ver.di. Abschnitt 5 untersucht einige der zentralen Voraussetzungen, auf denen die USamerikanische Kampagne basiert, und diskutiert, ob eine Übertragung auf deutsche Verhältnisse vorstellbar ist. Abschnitt 6 fasst die zentralen Ergebnisse zusammen.

\section{Die Organisationsgrundlagen der deutschen und der amerikanischen Gewerkschaften: ein kursorischer Vergleich}

Wolfgang Streeck argumentiert, dass sich nationale Systeme der industriellen Beziehungen hinsichtlich ihrer Organisationsgrundlagen unterscheiden lassen (1993: 40 ff.). Das Muster interessenpolitischer Organisierung wird demnach durch eine Konfiguration verschiedener Faktoren geprägt: die Identitäts- und Interessendefinitionen der Arbeitnehmer, die Organisationsformen dieser Interessen sowie die Institutionen, die den gesamten Prozess einbetten. Streeck argumentiert, dass sich Systeme der Arbeitsbeziehungen entlang zentraler Strukturgrundlagen entwickeln: Klasse, Beruf, Unternehmen oder Distrikt. Historisch war dabei nur selten jeweils eine Kategorie dominant. Zwar waren fast alle frühen Gewerkschaften Berufsgewerkschaften (z. B. die Drucker). Doch später koexistierten und konkurrierten in allen Ländern verschiedene Organisationsmodelle zumindest phasenweise. In den deutschen Arbeitsbeziehungen wurden die Klasse und das Unternehmen zum dominanten Organisationsfaktor, während das amerikanische System auf den Grundlagen berufs- und unternehmensbezogener Organisation basiert. Entlang dieser Merkmale haben sich die nationalen Gewerkschaften entwickelt.

\subsection{Deutschland}

Die klassenbasierte Interessenorganisation nimmt die gemeinsame Marktlage der Anbieter von Arbeitskraft als ihren Ausgangspunkt (vgl. im Folgenden ebd.: 41 f.). Dabei treten die professions- und unternehmensbezogenen Marktpositionen, Interessen und Identitäten von Arbeitnehmern zugunsten der Klassenidee in den Hintergrund. Die dieser Identitätsbestimmung entsprechende Organisationsform ist die Einheitsgewerkschaft. Zwar existiert in Deutschland keine diesem Idealtypus entsprechende allgemeine Gewerkschaft, doch kommen die Branchen- oder meist sogar Multi-Branchengewerkschaften dem Modell doch recht nah. Die Gewerkschaft ver.di ist dafür ein sehr gutes Beispiel. Sie erhebt den Anspruch, den gesamten öffentlichen und privaten Dienstleistungssektor zu organisieren und sowohl die Interessen der Straßenreiniger als auch die Belange der wissenschaftlichen Mitarbeiter an Hochschulen zu repräsentieren. ${ }^{1}$

Die relative Gleichheit der Einkommens- und Lebensverhältnisse ist für die Einheitsgewerkschaft die zentrale Norm der Entscheidungsfindung. Um dies durchzusetzen, sind

1 Dass empirisch auch die Einheitsgewerkschaft häufig von bestimmten Beschäftigtengruppen dominiert wird (bei ver.di ist dies z. B. der öffentliche Dienst), ändert nichts an dem gewerkschaftlichen Anspruch, eine übergreifende Interessenvertretung zu sein. Die Revitalisierungsbemühungen zielen ja gerade darauf $a b$, bisher unerschlossene Bereiche unter dem Dach der Einheitsgewerkschaft zu organisieren (z. B. den Handel). 
Einheitsgewerkschaften typischerweise niemals nur auf den Markt hin orientiert, sondern sie agieren gleichzeitig im politischen Raum. Eine marktnahe Interessenvertretungspolitik würde schnell an die Grenzen des „Marktwerts“ unterschiedlicher Arbeitnehmergruppen stoßen und damit die Zentrifugalkräfte stärken. Zudem sind klassenbasierte Einheitsgewerkschaften auf produktive Austauschprozesse mit dem Staat angewiesen. Wie bereits erwähnt, sind umfassend-inklusive Arbeitnehmerverbände nach Olson ohne staatliche Organisationshilfen kaum vorstellbar. Der Staat wiederum kann darauf hoffen, mit der Unterstützung zentralisierter und hoch integrierter Verbände auf die in ihnen getroffenen Entscheidungen Einfluss zu nehmen. Vor diesem Hintergrund tendieren Einheitsgewerkschaften im Spannungsfeld aus Mitgliedschafts- und Einflusslogik zum einflusslogischen Pol. Regelmäßig finden sie sich in korporatistischen Strukturen der Interessenvermittlung wieder, in denen die Verbände zu Teilnehmern an ,,privaten Interessenregierungen“" werden (Schmitter/Streeck 1999: 191 ff.). Diese sind Ausdruck eines „verbandlichen Modells sozialer Ordnung“. Die Gewerkschaften erfahren politische und institutionalisierte Anerkennung durch staatliche Akteure. Im Gegenzug richten sie ihre Politik nicht ausschließlich an den engen Kern ihrer Mitgliederinteressen aus. Der Begriff der „privaten Interessenregierung“ ist damit in zweierlei Hinsicht zu verstehen: Erstens beteiligen sich private Akteure an der Produktion verbindlicher politischer Entscheidungen. Zweitens regieren die Verbandsspitzen die Interessen ihrer Mitglieder, um gegenüber dem Staat die nötige Verpflichtungsfähigkeit sicherzustellen.

Vor diesem Hintergrund war der Einfluss der deutschen Gewerkschaften in der Vergangenheit deutlich stärker durch staatliche Anerkennung als durch die eigene Organisationsmacht bedingt. Ihr Organisationsvermögen war im internationalen Vergleich bestenfalls mäßig, und die Mitgliederentwicklung ist seit Dekaden rückläufig. Prognosen des DGB bis zum Jahr 2011 gehen davon aus, dass die Gewerkschaften dann seit der Wiedervereinigung $42 \%$ ihrer Mitglieder und $60 \%$ ihres Finanzvolumens verloren haben werden (Bremme/Fürniß/Meinecke 2007b). Die staatliche Anerkennung hatte auf die Arbeitnehmerverbände ambivalente Wirkungen. Einerseits wurden die Gewerkschaften relativ unabhängig vom Organisierungszwang, was wesentlich dazu beitrug, dass sie den kontinuierlichen Mitgliederverlust viele Jahre lang zwar zur Kenntnis nahmen, aber nicht ernsthaft bekämpften. Organisationsmacht war faktisch durch politische Institutionalisierung ersetzt worden (vgl. dazu auch Visser 1995: 53). Andererseits verstärkte die Institutionalisierung die Distanz zu den Beschäftigten, den kaum mehr plausibel gemacht werden konnte, dass sich eine auf Freiwilligkeit basierende individuelle Mitgliedschaft lohnt, wenn man doch auch als Trittbrettfahrer von den ausgehandelten Tarifverträgen profitieren kann.

Seit den 1990er Jahren wird durch den wachsenden Wettbewerbsdruck auf nationalen und internationalen Märkten der Betrieb zur konkurrierenden Bezugsgröße interessenpolitischer Organisierung. ${ }^{2}$ Institutionelle Grundlage dafür ist die Existenz von

2 Darüber hinaus ist seit einigen Jahren eine steigende Bedeutung von Berufsgewerkschaften $\mathrm{zu}$ beobachten, auf die in diesem Artikel jedoch nicht detailliert eingegangen werden kann. Von Bedeutung ist hier vor allem, dass die Zentrifugalkräfte gerade im Organisationsbereich der Einheitsgewerkschaft ver.di stärker werden, und zwar in verschiedene Richtungen gleichzeitig. 
Betriebsräten, die formal-rechtlich unabhängig von den Gewerkschaften sind und die eine stärkere Betriebszentrierung der Arbeitsbeziehungen häufig zumindest akzeptieren, wodurch eine deutliche Differenzierung der Tarifpolitik auf betrieblicher Ebene ermöglicht wird (Artus 2001). Auch im parteipolitischen Spektrum hat die Dezentralisierung viele Anhänger gefunden. Die SPD hat unter der Kanzlerschaft von Gerhard Schröder eine Flexibilisierung und Dezentralisierung der Tarifpolitik gefordert. Dies gilt noch ausgeprägter für die CDU/CSU, die mit Plänen zur betriebszentrierten Reform des Arbeitsrechts in den Bundestagswahlkampf 2005 zog. Dies hatte unmittelbare Folgen für die Gewerkschaften. Die DGB-Gewerkschaften konnten sich lange auf ihre Eingebundenheit in die politischen Entscheidungsprozesse verlassen. Dass sie sich heute für das amerikanische Mobilisierungs- und Organisierungsmodell interessieren, liegt auch am Verlust ihrer staatlichen Anerkennung, wodurch sie auf ihre Organisationsmacht zurückgeworfen werden.

\subsection{USA}

In den USA hat die Kategorie der Klasse historisch nie eine große Rolle gespielt. Die frühen US-Gewerkschaften waren Berufsgewerkschaften. Mit der rechtlichen Verankerung des Tarifgedankens im Rahmen des „National Labor Relations Act“ 1937 wurde der Betrieb zum dominanten Prinzip interessenpolitischer Organisation (Katz/Kochan 2000: 36 ff.). Aufgrund der fragmentierten Entscheidungsstrukturen im politischen System der USA koexistieren verschiedene Regelungen im Bereich der Arbeitsbeziehungen, die im Wesentlichen auf bundesstaatlicher Basis gestaltet werden. Generell gilt, dass die Anerkennung einer Gewerkschaft als Interessenvertretung an das Votum der Beschäftigten in einer zuvor definierten Organisationseinheit (meist der Betrieb) gebunden ist. Dabei stehen zwei Verfahren zur Verfügung. Im für eine Gewerkschaft günstigeren Fall wirbt sie per Unterschriftensammlung um Unterstützung im Betrieb. Bekunden mindestens $50 \%$ der Beschäftigten ihre Zustimmung und erkennt das Management auf dieser Basis die Gewerkschaft als Verhandlungspartei an, ist sie als Interessenvertretung legitimiert (card-check Verfahren). Im ungünstigeren Fall muss sie formale Anerkennungswahlen gewinnen. Gewinnt sie diese Wahl, wird sie unabhängig von der Zustimmung der Arbeitgeberseite als Interessenvertretung eingesetzt (Dribbusch 2007: 27). Beide Verfahren implizieren die Möglichkeit, dass einige Betriebe eines Unternehmens gewerkschaftlich organisiert sein können und andere nicht. In einigen Bundesstaaten wurden Schutzklauseln verabschiedet, um die Gewerkschaften vor Trittbrettfahrern zu schützen. Ein Betrieb kann zum „union shop“ erklärt werden, was bedeutet, dass die Beschäftigten im Falle der gewerkschaftlichen Anerkennung automatisch Mitglied werden oder aber eine Gebühr entrichten, was einer Quasi-Zwangsmitgliedschaft entspricht.

Zwei Unterschiede zum deutschen System sind hier von großer Bedeutung. Während erstens im deutschen System staatliche Organisationshilfen gewerkschaftliche Organisationsmacht tendenziell ersetzt haben, werden sie im amerikanischen System nur dann gewährt, wenn die Gewerkschaft die Legitimation durch die Beschäftigten nachgewiesen hat, und zwar in jedem einzelnen Betrieb (Visser 1995: 53). Zweitens existiert in den USA ein direkter Kausalzusammenhang zwischen Organisation und Einfluss: Amerikanischen Gewerkschaften wird nur dann Einfluss (Tarifverhandlungen) gewährt, 
wenn sie zuvor die Beschäftigten für sich gewonnen haben. Anders herum haben sie in vielen Staaten durch erfolgreiche Anerkennungswahlen im Rahmen von „union shop“Abkommen nicht nur Einfluss, sondern auch neue Mitglieder gewonnen (und zwar auch diejenigen Beschäftigten, die zuvor gegen eine gewerkschaftliche Interessenvertretung gestimmt hatten). Im deutschen Fall sind Organisation und Einfluss nur lose gekoppelt. Wenn die Gewerkschaften Tarifverträge oder Betriebsräte durchsetzen, heißt dies noch nicht, dass sie auch neue Mitglieder gewinnen, weil die Mitgliedschaft auf freiwilliger Basis beruht, Betriebsräte formal unabhängig sind und tarifliche Regelungen von den Arbeitgebern meist auch auf Nicht-Mitglieder ausgedehnt werden.

Der Zwang, Anerkennungswahlen zu gewinnen, ist für die US-Gewerkschaften eine starke Organisationsrestriktion. Sie müssen nachweisen, dass $30 \%$ der Beschäftigten eines Betriebs Anerkennungswahlen befürworten, bevor eine Wahl angesetzt wird (Katz/Kochan 2000: 150). Während des Wahlkampfes steht es der Arbeitgeberseite frei, eine Gegenkampagne zu initiieren. Das sogenannte ,union-busting“ zielt darauf ab, den Wahlkampf durch aggressive Methoden und den Einsatz spezialisierter Anwälte zugunsten des Arbeitgebers zu beeinflussen (Levitt 1993). Wurden 1964 noch $61 \%$ aller Anerkennungswahlen gewonnen, trifft dies heute nur noch auf etwa $50 \%$ der Fälle zu (Raffo 2007: 179). Aufgrund der aggressiven Gegenstrategien der Arbeitgeberseite treten die US-Gewerkschaften meist gar nicht erst zur Wahl an, wenn sie nicht mindestens 65 bis $70 \%$ der Beschäftigten hinter sich wissen.

\section{Zentrale Komponenten der Revitalisierungsstrategien}

Für den Vergleich ist von zentraler Bedeutung, dass die amerikanischen Gewerkschaften immer schon viel stärker als die deutschen Schwesterverbände auf die Mobilisierung von Input-Legitimität angewiesen waren. Damit ist gemeint, dass das Repräsentationsmandat aus den Präferenzen der Basis abgeleitet wird. Ohne die aktive Zustimmung der Beschäftigten gibt es keine gewerkschaftliche Interessenvertretung. Dadurch erklären sich die Strategien der offensiven Mitgliederwerbung und -beteiligung. Die deutschen Gewerkschaften sind bisher durch ihren hohen Institutionalisierungsgrad tendenziell weniger von Input-Legitimität abhängig gewesen. Allenfalls mussten sie damit rechnen, von den Mitgliedern an der Output-Legitimität, also an den erzielten Resultaten der Interessenvertretung (z.B. Lohnabschlüssen) gemessen zu werden (zum Begriffspaar Input- und Output-Legitimität vgl. Scharpf 1970).

Wenn die deutschen Gewerkschaften heute amerikanische Strategien adaptieren, kann dies auch als ein (zumindest partieller) Wechsel von der Output- zur Input-Legitimierung interpretiert werden, der gegenwärtig auch bei Betriebsräten zu beobachten ist (Rehder 2006). Seit Jahren befinden sich die Gewerkschaften in Abwehrkämpfen, um die einstmals erzielten Standards zu halten. Häufig gelingt dies nur bedingt; die Output-Legitimität ist prekär geworden. Weil unter den Bedingungen des intensivierten Wettbewerbs kaum damit zu rechnen ist, dass sich dies in absehbarer Zukunft ändert, brauchen die Gewerkschaften eine direkte Bindung an ihre Mitglieder, um ihre Organisation zu stabilisieren und ihre Politik zu legitimieren. Aus diesem Grund liegt der Kern aller Revitalisierungsbemühungen in der Mobilisierung. Nicht selten wird dabei 
sogar vom Ziel der Demokratisierung gesprochen (Schreieder 2007: 155). Und es ist bemerkenswert, dass zumindest einige der deutschen Gewerkschaften angesichts ihrer existenziellen Bedrohung (zumindest rhetorisch) ihr Heil in der Demokratie suchen.

Die Revitalisierungsversuche basieren nicht auf einer Theorie. Was als „OrganizingModell“ bezeichnet wird, ist nicht mehr als ein strategischer Instrumentenkasten, aus dem sich die Organisationen bedienen. Einige Praktiken lassen sich als konstitutiv für die Revitalisierungsversuche identifizieren (Dribbusch 2007: 31 ff.).

Als zentrales Instrument dienen strategische Kampagnen gegen gewerkschaftsfeindliche Unternehmen. Gewerkschaftliche Aktionsformen erschöpfen sich nicht mehr nur in Streiks und Demonstrationen. Vielmehr wird versucht, durch strategisch inszenierte Kampagnen den öffentlichen Raum zu besetzen. Dabei kommen Taktiken zur Anwendung, die zum Teil sehr erfolgreich von Nichtregierungsorganisationen praktiziert werden. Amnesty International z. B. übt seit Jahrzehnten durch Postkartenaktionen Druck auf autoritäre Regime aus, um die Freilassung politischer Gefangener zu erreichen. Das Berufsbild des „Campaigners“, das von Organisationen wie Greenpeace bekannt ist, findet nun auch seinen Platz in den Gewerkschaften und unterscheidet sich stark von dem des typischen Gewerkschaftsfunktionärs.

Um Kampagnen durchzuführen, sind intensive Recherche- und Planungsaktivitäten nötig. Heute beschäftigt die SEIU rund 200 Analysten, um Märkte und Unternehmen zu beobachten und Strategien zu entwerfen (Woodruff 2007: 111). Die Aktivitäten basieren auf der Idee, zu Beginn ein einzelnes Unternehmen zu attackieren und den Aktionsradius schrittweise zu erweitern. Unternehmen werden als gesellschaftlich eingebettete soziale Institutionen betrachtet, die durch vielfache Bezüge in eine Umwelt integriert sind, z. B. durch Beziehungen zu Kunden, Investoren, Geschäftspartnern, politischen Agenturen etc. (Greven 2006: 255). In den „Forschungsabteilungen“ einer Gewerkschaft werden die Interdependenzen von Unternehmen analysiert, um erstens geeignete Zielunternehmen zu sondieren und zweitens Schlüsselverbindungen des Unternehmens zu seiner Umwelt zu finden, um auch diese zu attackieren. Die Gewerkschaften beschränken sich also nicht nur darauf, ein Unternehmen anzugreifen, sondern sie stellen darauf $a b$, dessen Umwelt für ihre Zwecke zu mobilisieren.

Sogenannte Organizer, ,die auf Grund von Ausbildung und Erfahrung in der Lage sind, Beschäftigte, die interessiert sind, gewerkschaftlich aktiv zu werden, ausfindig zu machen, zu motivieren und zur Selbsttätigkeit anzuleiten“ (Dribbusch 2007: 32), sind die Hauptakteure der Kampagnen. Lead-Organizer sollen die Teams anleiten. Darüber hinaus wird versucht, Mitglieder als Freiwillige für die externen Mobilisierungskampagnen zu gewinnen, um das hauptamtliche Personal zu unterstützen (Nissen 1998: 135 f.). Hier wird eine gewisse Nähe zu sozialen Bewegungen besonders deutlich: „Soziale Bewegungen existieren in der Regel nicht ohne Organisation, Organisation ist aber nicht das Entscheidende an der Bewegung“ (Raschke 1985: 80). Gewerkschaften im Revitalisierungsprozess agieren nicht nur durch ihre Funktionäre. Vielmehr kommt die personalintensive Strategie nicht ohne die Aktivität der individuellen Mitglieder aus. Um die nötigen Qualifikationen zu erwerben, werden die Organizing-Teams speziell ausgebildet.

Die neuen Strategien basieren sehr stark auf direkten Aktionsformen: Demonstrationen, das Tragen von Emblemen, Pressekonferenzen, Straßenblockaden, Aufrufe zu 
Protesten und Boykotts, ziviler Ungehorsam - der Phantasie sind keine Grenzen gesetzt. Dabei wird mit steigenden Eskalationsstufen gearbeitet. Daraus folgt, dass auch der Verlauf einer Kampagne strategisch geplant wird. Um durch die Aktionen hinreichend Druck aufzubauen, werden Koalitionen mit anderen politischen und sozialen Gruppierungen gebildet. Bevorzugte Koalitionspartner sind Nichtregierungsorganisationen oder soziale Bewegungen, aus deren Kreisen in den USA auch ein Teil der „neuen“ Gewerkschaftsfunktionäre rekrutiert wird. Darüber hinaus sind lokale Organisationen, z. B. aus dem Wohlfahrtsbereich, religiöse Gruppen sowie lokale Persönlichkeiten und Politiker, von Bedeutung.

Aus dem Gesagten geht hervor, dass die Kampagnen sowohl starke Top-Down-Elemente als auch Aspekte der Bottom-Up-Orientierung aufweisen. Je nachdem, welche Seite man betonen mag, kommt man zu anderen Bezeichnungen. Diejenigen, die das Phänomen gern als „Social Movement Unionism“ charakterisieren, betonen den Bottom-Up-Aspekt, also die Mobilisierung der Mitglieder, die Koalitionsbildung zwischen den Gewerkschaften mit anderen Gruppen und die Wiederbelebung als soziale Basisbewegung. Diejenigen, die eher von „Organizing“ sprechen, beschäftigen sich vor allem mit der strategischen Planung durch die Verbandszentralen.

Empirisch geht mit der Neuorientierung eine deutliche Umwidmung der Organisationsressourcen einher. In den USA leistete die Dachorganisation AFL-CIO rund 20 Millionen Dollar Anschubfinanzierung, um die Einrichtung von lokalen „organizing departments“ zu initiieren (Bronfenbrenner et al. 1998: 1). Finanzielle Mittel (etwa ein Drittel des Etats) werden vor Ort von der Administration und Betreuung der Mitglieder abgezogen und in die Finanzierung politischer Kampagnen zur Rekrutierung von Mitgliedern und zur Durchsetzung der Organisationsinteressen investiert. Auch die Auswahl und Ausbildung der Funktionäre folgt diesem Primat (Milkman 2006: 27 ff.). Das heißt, bei der Mobilisierung der Mitglieder wird aus der Not eine Tugend gemacht. Wenn nennenswerte Ressourcen, die bisher in Serviceangebote investiert wurden, nun in die Kampagnenpolitik umgelenkt werden, resultiert daraus der Zwang, dass die Mitglieder ihre Probleme vor Ort stärker als bisher selbst lösen. Auch in diesem Sinne ist also eine Einbindung der Mitgliedschaft in die Revitalisierungsstrategien notwendig.

\section{Fallbeispiele: Revitalisierung in den USA und Deutschland}

Die Komponenten der Revitalisierungsstrategie und ihre Funktionsweise sollen im Folgenden anhand von zwei Fallbeispielen illustriert werden, der „Justice for Janitors“Kampagne in Los Angeles und der „Lidl“"-Aktion von ver.di.

\subsection{Justice for Janitors}

Die meistzitierte Kampagne amerikanischer Gewerkschaften ist die „Justice for Janitors"-Aktion, die in verschiedenen US-amerikanischen Städten mit Erfolg durchgeführt wurde (vgl. Waldinger et al. 1998; Milkman/Wong 2001; Rudy 2004; Milkman 2006). Das Ziel bestand darin, das Personal lokal ansässiger Reinigungsfirmen zu organisieren und Tarifverträge durchzusetzen. Die Beschäftigten arbeiten in dieser Branche 
unter sehr schlechten Arbeitsbedingungen, die häufig nicht einmal den gesetzlichen Mindestregelungen entsprechen. Überwiegend sind sie (zumeist illegale) Immigranten lateinamerikanischer Herkunft. Die Prominenz dieser Kampagne basiert auch darauf, dass hier eine Arbeitnehmergruppe erreicht werden konnte, von der man angesichts ihrer Marktlage die Bereitschaft zum kollektiven Handeln nicht erwartet hätte.

Die Kampagne wurde zentral von der Gewerkschaftsführung geplant und lokal in verschiedenen US-amerikanischen Städten implementiert. Die Auswahl der Branche, der zu attackierenden Unternehmen und der angewendeten Taktiken erfolgte nicht zufällig, sondern sie war das Ergebnis intensiver Recherche und Planung. Das Reinigungswesen wurde ausgewählt, weil es hundertprozentig standortgebunden ist: Ein Gebäude kann nur dort gereinigt werden, wo es steht. Der Arbeitgeber kann sich dem von der Gewerkschaft ausgeübten Druck also nicht durch Standortverlagerung entziehen. Gleichzeitig ist das Reinigungsgewerbe in vielen US-amerikanischen Städten hoch konzentriert. In Los Angeles, wo die Kampagne besonders erfolgreich war, dominierten Mitte der 1990er Jahre zwei große Unternehmen den Markt. Eine erfolgreiche Kampagne gegen diese beiden Unternehmen würde bedeuten, einen erheblichen Anteil des Markts zu organisieren (Erickson et al. 2008: 5).

Typischerweise zielen die Unternehmenskampagnen nicht darauf ab, einem Unternehmen ökonomischen Schaden zuzufügen (z. B. durch Streiks). Vielmehr geht es darum, politischen Druck aufzubauen. Dabei wird häufig das adressierte Unternehmen nicht direkt angegriffen, sondern indirekt, indem z. B. die Geschäftspartner in den Konflikt einbezogen werden. Das Arbeitgeberunternehmen zu höheren Löhnen zu zwingen, würde nur bedeuten, seine Marktchancen zu verschlechtern. Deshalb rücken die eigentlichen Auftraggeber ins Zentrum des gewerkschaftlichen Interesses. Die Annahme war, dass sich das Reinigungsgewerbe für eine Kampagne sehr gut eignen würde, weil zwischen den Reinigungsfirmen und ihren Auftraggebern sensible Vertrauensbeziehungen existieren. Die Reinigungskräfte haben über Nacht einen unkontrollierten Zugang zu den Räumen und damit zum Eigentum der Auftraggeber.

Die Gewerkschaft SEIU nutzte für sich den Umstand, dass in den attackierten Unternehmen sowohl gewerkschaftlich organisierte als auch nicht organisierte Niederlassungen koexistierten. Den organisierten Betrieben bot sie günstigere Tarifverträge an, damit sie in der Konkurrenz mit den nicht organisierten Betrieben bestehen konnten und sich während der Kampagne nicht mit den angegriffenen Betrieben solidarisierten. Dabei wurden Hintergrundvereinbarungen getroffen, die besagten, dass zum alten Lohnniveau zurückgekehrt werde, wenn die Organisationsbemühungen in den anderen Zweigstellen erfolgreich verlaufen. Als zweite Säule wurden traditionelle Werbeinstrumente angewendet. Zudem wurde das Rechtssystem intensiv bemüht. Arbeitgeber, die Mindeststandards ignorierten, wurden verklagt.

Die eigentliche Kampagne dient dazu, öffentlichen Druck aufzubauen. Er wird multipliziert, indem die Umwelt des Unternehmens einbezogen wird, und zwar durch direkte Aktion. Zum Beispiel werden Geschäftspartner beim Lunch im Restaurant oder beim Sport im Golfclub oder im Kino ,besucht“, um das Unternehmen und seine Geschäftspartner gegenüber der Öffentlichkeit zu diskreditieren. „Blaming“ und „shaming“ sind wichtige Bestandteile der Kampagnen. Im Fall der Janitors in Los Angeles wurden sehr effektiv die Mieter der Gebäude, in denen die Reinigungsfirmen tätig waren, attackiert. 
Zum Beispiel wurden die Foyers besetzt oder an fünf Tagen pro Woche die Fahrstühle blockiert, sodass die Mieter ihre Apartments oder Büros nur zu Fuß erreichen konnten, auch wenn sich diese im 17. Stock befanden. Hilfreich war auch, dass einige Geschäftspartner prominente Namen hatten, wodurch die öffentliche Aufmerksamkeit erhöht wurde. Politische Akteure wurden z.B. durch Straßenblockaden zu Hauptverkehrszeiten mobilisiert. Die Strategie, die Aufmerksamkeit der Politik auf sich zu ziehen, war vor allem in Wahlkampfzeiten sehr erfolgreich.

Die grundlegende Idee besteht darin, lokal politische Krisen zu erzeugen, die andere Akteure zum Handeln zwingen. Dabei ist es hilfreich, wenn die Gewerkschaft an bestehende Konfliktlinien andocken und dadurch politische Koalitionen mit anderen Gruppen bilden kann. Wenn etwa in einer Kommune ein Konflikt über sozialen Wohnungsbau ausgetragen wird, eröffnet dies den Gewerkschaften die Option, ihre sozialen Fragen im Windschatten der bereits geführten Debatte einzuspeisen. Eine wichtige Rolle spielt dabei die mediale Aufmerksamkeit. Die SEIU setzte den von ihr angestrebten Tarifvertrag in Los Angeles durch, nachdem auf einer Protestveranstaltung einige Teilnehmer (darunter auch eine schwangere Frau) von Polizisten vor laufender Fernsehkamera misshandelt worden waren, was die lokalen Behörden zum Handeln zwang. Dieses Beispiel zeigt auch, dass die Kampagnen mit einem erheblichen Maß an Militanz kalkulieren müssen, um hinreichend Druck zu erzeugen. Einige amerikanische Autoren sprechen in diesem Zusammenhang auch von Guerilla-Taktiken (Waldinger et al. 1998: 115).

Aus den skizzierten Elementen der „Justice for Janitors“-Initiative wird deutlich, wie ressourcenabhängig die strategischen Kampagnen sind. Über einen Zeitraum von zwei Jahren mehrmals pro Woche an verschiedenen Stellen Aktionen durchzuführen, erfordert einen hohen Personaleinsatz. Vor diesem Hintergrund ist es für die Gewerkschaften unverzichtbar, die Mitglieder zur Teilnahme an derartigen Kampagnen zu mobilisieren. Trotz dieser Schwierigkeiten ist es der SEIU gelungen, die Erfolge auf Dauer zu stellen. In Los Angeles wurden bereits mehrere Tarifverträge abgeschlossen, die eine schrittweise Verbesserung der Arbeitsbedingungen bewirkten. Bundesweit hat die SEIU mit der neuen Strategie zwischen 1996 und 2006 mehr als 650.000 neue Mitglieder rekrutiert (Woodruff 2007).

\section{2 „Lidl“-Kampagne}

Die deutsche Dienstleistungsgewerkschaft ver.di hat damit begonnen, schrittweise einen Lernprozess $\mathrm{zu}$ initiieren, in dem zumindest einige Komponenten der amerikanischen Strategie adaptiert werden sollen (Schreieder 2005, 2007). Dieser Prozess ist in der Organisation weder institutionalisiert noch unumstritten. Häufig wird er sogar nur von einzelnen Personen getragen. „Campaigner“ und „Organizer“" werden durch Externe oder bestenfalls prekär Beschäftigte gestellt. Doch der Vorsitzende Frank Bsirske gilt als ein starker Befürworter der neuen Strategie (Bsirske 2005; zu der Haltung verschiedener Gewerkschaften vgl. Greven 2006: 259 ff.). Von zentraler Bedeutung für den Lernprozess sind gemeinsame Treffen amerikanischer und deutscher Gewerkschaftsmitarbeiter, in denen Erfahrungen ausgetauscht werden. So fand u. a. im April 2005 in Hamburg eine „Organizing-Woche“ statt (ver.di 2005). Der Austausch wird dadurch verstärkt, dass einzelne deutsche Funktionäre an amerikanischen „Trainingscamps“ 
teilnehmen. Der Landesbezirk Hamburg zielt darauf ab, eines der gewerkschaftlichen Bildungszentren als „Kompetenzzentrum für Organizing“ zu etablieren. Darüber hinaus wird angestrebt, ein Drittel der (personellen) Ressourcen für Organisierungs- und Kampagnenzwecke einzusetzen.

Ver.di lernt nicht nur durch den Erfahrungsaustausch, sondern auch dadurch, dass US-Gewerkschafter als „Lehrmeister“ an deutschen Kampagnen teilnehmen (Greven 2006: 265). Von besonderer Bedeutung ist dabei das Netzwerk OrKa (Organisierung \& Kampagnen), das unter Beteiligung deutscher und amerikanischer Mitarbeiter als Kampagnenagentur maßgeblich an der Vorbereitung entsprechender Aktivitäten beteiligt ist. Zudem wurde ein Internetportal (www.neverworkalone.org) eingerichtet, um eine bessere Vernetzung und Koordinierung der Aktionen zu ermöglichen.

Getestet wurde das amerikanische Revitalisierungskonzept z. B. mit einer Kampagne im Hamburger Sicherheitsgewerbe (Bremme 2007). Breite Aufmerksamkeit erlangte es jedoch erst mit der Kampagne gegen die Supermarktkette „Lidl“ (seit 2004), die darauf abzielte, die Etablierung von Betriebsräten zu erreichen. Der Schwarz-Konzern behindert die Gründung von Betriebsräten seit jeher massiv. Diese sind für die Gewerkschaften aber zur Rekrutierung von Mitgliedern von zentraler Bedeutung, da sie als betriebliche Interessenvertretung im täglichen Kontakt mit den Beschäftigten stehen (Goerke/Pannenberg 2007). Für die Mitgliederrekrutierung sind sie jedoch nur dann nützlich, wenn die formal-rechtlich unabhängigen Betriebsräte selbst gewerkschaftlich organisiert sind. Hier zeigt sich bereits, wie sehr die Entkopplung von Einfluss und Organisationsmacht erfolgreiche Kampagnen amerikanischer Art behindert. Zwar kann ver.di versuchen, die Unternehmensleitung von „Lidl“ dazu zu bewegen, die Einrichtung von Betriebsräten nicht länger zu behindern. Dies bedeutet jedoch nicht, dass dann auch Betriebsräte gewählt werden oder wenn ja, sich diese gewerkschaftlich organisieren. Die Kausalkette zwischen Kampagne und Erfolg ist deutlich stärker durchbrochen und kann nur mittelbar strategisch beeinflusst werden.

Die zentralen Elemente der amerikanischen Revitalisierungsstrategie finden sich auch in der „Lidl“'-Kampagne wieder. Die Kampagne basierte auf verschiedenen Säulen (vgl. Rickens 2006; Schreieder 2007), wobei - ähnlich wie bei den amerikanischen Vorbildprojekten - die Kampagnenplanung in der Zentrale vorgenommen wurde. In lokalen „Aktivitätszentren“ auf Bezirksebene fanden die eigentlichen Organisierungsbemühungen statt. Dabei kam erstens der Mitgliedermobilisierung ein großer Stellenwert zu. Im Rahmen der sogenannten „Lidl-Filialbegleitung“ sollten speziell geschulte Gewerkschaftsmitglieder die Beschäftigten ansprechen und sie ermutigen, ihre Interessen zu vertreten. Zweitens wurden die Gewerkschaftsmitglieder in ihrer Identität als Verbraucher angesprochen. Die direkte Aktion spielte auch hier eine wichtige Rolle. Drei Millionen Postkarten wurden versandt, mit denen die Mitglieder in den Filialen gegen die Behinderung der Interessenvertretungsarbeit protestieren sollten. Drittens wurden die Medien genutzt. Die Erfahrungsberichte vieler Mitarbeiter wurden in einem sogenannten „Schwarzbuch Lidl“ zusammengefasst und veröffentlicht. Ein von ver.di eingerichtetes Internetforum diente als Plattform für Mitarbeiter, um sich anonym über ihre (schlechten) Erfahrungen mit dem gemeinsamen Arbeitgeber auszutauschen. Und viertens wurde eine (lockere) Koalition mit verschiedenen Akteuren geschlossen, darunter Umweltschutzverbände, ökologisch orientierte Bauernverbände und vor allem 
das globalisierungskritische Netzwerk „attac“, um gemeinsame Demonstrationen oder Blockadeveranstaltungen durchzuführen. Die Koalitionen beruhen auf dem Prinzip „Gemeinsam marschieren - getrennt schlagen“, da jede Gruppe ihre eigenen Ziele verfolgt. Während ver.di mit den genannten Maßnahmen die Einrichtung von Betriebsräten erreichen wollte, blockierte die ökologisch orientierte Arbeitsgemeinschaft bäuerliche Landwirtschaft die „Lidl“-Zentrale mit einer Kuh, um gegen das Preisdumping des Unternehmens bei Milchprodukten zu protestieren. Andere Organisationen setzten sich für den Vertrieb fair gehandelter Produkte aus Entwicklungsländern (z. B. Bananen) ein.

Gemeinsam war den Koalitionären also im Wesentlichen die Gegnerschaft zum Unternehmen. Dies war hilfreich für die Erregung medialer Aufmerksamkeit und ermöglichte den wechselseitigen Zugriff auf Ressourcen. Es bot dem Unternehmen jedoch auch die Option, aus der Fülle der unterschiedlichen Forderungen einige zu selektieren, um andere abzuwehren. Dabei hat man vor allem auf Verbraucherinteressen reagiert. So hat „Lidl“ akzeptiert, den Wachstumsmarkt der ökologischen und fair gehandelten Produkte ins Sortiment zu integrieren. Betriebsräte existieren aber immer noch sehr selten. Für die Gewerkschaft ist die Erfolgsbilanz also sehr durchwachsen, auch wenn einige Hundert der 40.000 „Lidl“-Beschäftigten in Deutschland als Mitglieder gewonnen werden konnten. Auch daran wird deutlich, dass für deutsche Gewerkschaften der Weg von der Kampagne zum Organisationserfolg lang ist.

Ähnlich wie die amerikanische „Janitors“-Kampagne wurde auch das „Lidl“-Projekt strategisch geplant. Dennoch ist offenkundig, dass einige der Faktoren, die den amerikanischen Erfolg zu erklären helfen, im deutschen Fall nicht vorlagen. Zwar sind auch Supermärkte standortgebunden; dies gilt jedoch nicht so unbedingt wie im Reinigungsgewerbe. So musste ver.di die Erfahrung machen, dass „Lidl“ lieber einen Supermarkt schließ - um ihn an anderer Stelle neu zu eröffnen - als einen Betriebsrat zuzulassen. Auch die Kunden sind weniger ortsfest als im Reinigungsgewerbe. Zudem ist die Branchenstruktur im Handel sehr fragmentiert. Auch wenn der Schwarz-Konzern zweifellos ein erhebliches Gewicht hat, liegt hier kein mit dem US-Vorbild vergleichbarer Konzentrationsgrad vor, wo zwei attackierte Unternehmen einen großen Teil des Marktes abdecken und damit auch für die Kunden nur wenige attraktive Möglichkeiten bestehen, sich dem gewerkschaftlichen Druck zu entziehen.

Doch selbst ohne diese strategischen Probleme stößt das amerikanische Konzept in Deutschland an grundsätzliche Grenzen. Sie bilden das Thema des folgenden Abschnitts.

\section{Grundlagen US-amerikanischer Erfolge und ihre Übertragbarkeit auf Deutschland}

Auch wenn in der Literatur die Bedeutung der Strategiefähigkeit von Organisationen hervorgehoben wird, kann kein Zweifel daran bestehen, dass jede gewerkschaftliche Erneuerungsstrategie in institutionelle, strukturelle und normative Bedingungen eingebettet ist, die die Entwicklung und Wirkung einer Strategie beeinflussen. Die Übertragung kontextgebundener Strategien in fremde institutionelle Systeme kann als technisches Problem diskutiert werden. Im Zentrum steht dann die Frage: Würde diese Strategie 
auch bei den deutschen Gewerkschaften funktionieren? Man kann die Debatte aber auch normativ akzentuieren. Die Frage würde dann lauten: Sollen die Gewerkschaften diese Strategie wirklich wollen? Beide Aspekte stehen natürlich in einem engen Zusammenhang, denn einerseits sollten um ihr Überleben kämpfende Organisationen nur das wollen, was sie auch können. Andererseits ist fraglich, ob sie Dinge tun sollen, nur weil sie es können. Einige kritische Punkte werden im Folgenden angesprochen.

\subsection{Voraussetzungen des Organisationslernens}

Es wurde bereits darauf hingewiesen, dass der gewerkschaftliche Strategiewechsel eine Abkehr vom institutionell scheinbar vorgegebenen Pfad darstellt. Die Organisationssoziologie beschäftigt sich seit einiger Zeit mit der Frage, wann bzw. unter welchen Bedingungen strategisches Handeln, das von organisational ausgebildeten Routinen abweicht, erwartbar ist. Gern wird dabei auf die Figur des institutionellen Unternehmers zurückgegriffen (DiMaggio 1988: 15). Ein Unternehmer in diesem Sinne ist nach DiMaggio ein Akteur, der ein Interesse am Wandel einer Organisation hat und über die Ressourcen verfügt, dieses Interesse auch durchzusetzen (ebd.). Dabei muss er gegenüber der Umwelt, vor allem aber auch innerhalb der Organisation, Legitimitätsreserven für den Wandel mobilisieren sowie Machtstrukturen in Rechnung stellen (Beckert 1999: 791 f.). Wo mächtige oppositionelle Akteure existieren und keine Akzeptanz für den Wandel herbeigeführt werden kann, wird ein Handeln abseits des institutionell vorstrukturierten Pfades unwahrscheinlich.

Die Forschung über die Spielarten des Kapitalismus hat gezeigt, dass die Fähigkeit von Organisationen (hier: Unternehmen) zur radikalen Innovation aufgrund ihrer institutionellen Einbettung sehr unterschiedlich verteilt ist (Hall/Soskice 2001b: 39 f.). Unter anderem erweitern hierarchische Entscheidungsstrukturen, flexiblere Arbeitsmärkte und ein geringer Kündigungsschutz in der liberalen amerikanischen Ökonomie den Spielraum für radikale Innovationen, weil ein Strategiewechsel durch Personalaustausch forciert werden kann. Die Organisation kann lernen, ohne auf individuelle Lernprozesse angewiesen zu sein. In der koordinierten deutschen Ökonomie dominiert nach Hall und Soskice demgegenüber die inkrementelle Innovation, weil Beschäftigungssicherheit und eine starke Veto-Position der Beschäftigten radikalen Wandel eher bremsen.

Wendet man diese Überlegungen auf die deutschen und amerikanischen Gewerkschaften an, wird deutlich, dass das Abweichen von etablierten Routinen für die US-Gewerkschaften weniger schwierig war, als es für die deutschen Gewerkschaften sein wird. Die Forschung über die Revitalisierung der US-Gewerkschaften hat deutlich gemacht, dass die strategische Neuorientierung mit einem erheblichen strukturellen Organisationswandel einherging. Die externe Rekrutierung neuer Führungskräfte mit Erfahrungen im Milieu sozialer Bewegungen, die die Rolle des „institutionellen Unternehmers“ übernehmen, gilt als eine zentrale Erfolgsvariable der Revitalisierung (Voss/Sherman 2000).

Im amerikanischen Fall wird John Sweeney diese Rolle zugeschrieben, der sowohl in der SEIU als auch in der AFL-CIO maßgeblich für den Strategiewechsel verantwortlich war (Waldinger et al. 1998: 109). Sweeney erhöhte den im Vergleich zu anderen US-Gewerkschaften niedrigen Gewerkschaftsbeitrag der SEIU. Mit den gewonnenen Mitteln 
baute er den ehemals schlanken Personalbestand an der Spitze seines Verbandes aus, um hauptamtliche Organizer und Analysten einstellen zu können. Er rekrutierte das neue Personal überwiegend aus dem Umfeld der neuen sozialen Bewegungen. Damit schuf er Parallelstrukturen zur bestehenden Organisation, um die neue Strategie zu realisieren. Auf lokaler Ebene fand zudem in gewissem Umfang ein Personalaustausch statt. Diejenigen Organisationseinheiten, die sich der neuen Mobilisierungsstrategie verschrieben hatten, stellten die Beschäftigten vor die Wahl: „change or leave“ (Fletcher/Hurd 1998: 42). Zudem wurden neue Anreizstrukturen für hauptamtliche Organizer geschaffen, wie z. B. eine bessere Bezahlung und Aufstiegsmöglichkeiten (Woodruff 2007: $100 \mathrm{f}$.).

Die SEIU hat bei der Entwicklung ihres Revitalisierungskonzepts also in erheblichem Maße von dem institutionellen Umfeld profitiert, das sie bei Unternehmen gerade bekämpft: nämlich hierarchische Entscheidungsstrukturen und einen flexiblen Arbeitsmarkt als Drohpotenzial und/oder Puffer der Personalpolitik. Diese Praktiken stehen für deutsche Gewerkschaften nicht so ohne Weiteres zur Verfügung. Der Funktionärsapparat ist im Vergleich zu den amerikanischen Schwesterverbänden bereits groß. Eine Erhöhung des Mitgliederbeitrags durch einzelne Gewerkschaften widerspräche dem Prinzip der Einheitsgewerkschaft, das sich auch in einem einheitlichen Mitgliedsbeitrag für alle DGB-Gewerkschaften ausdrückt. Die Strategie der Schaffung von Parallelstrukturen, die einen potenziellen Widerstand zu überwinden helfen, ist also durch Ressourcenknappheit begrenzt. Auch werden die internen Entgeltsysteme und Beförderungsstrukturen nicht ohne Weiteres per hierarchischer Entscheidung zu verändern sein, weil es sich hierbei teilweise um mitbestimmungspflichtige Tatbestände handelt.

Zusammengefasst: Die US-Verbände konnten teilweise auf der Makroebene lernen, ohne auf individuelle Lernprozesse angewiesen zu sein. Hier konnte Wandel durch Personalaustausch bzw. durch Personalausbau durchgesetzt werden. Die deutschen Gewerkschaften hingegen sind in höherem Maße auf einen deutlich anspruchsvolleren Mechanismus des Organisationslernens angewiesen, der auf individuellen Lernprozessen innerhalb der Gewerkschaft basiert. Potenzielle interne Veto-Spieler können nicht ohne Weiteres ausgeschaltet oder umgangen werden. Vielmehr müssen sie überzeugt werden. Die Skepsis bezüglich der amerikanischen Strategie ist jedoch groß, weil die Militanz der Kampagnen bei den sozialpartnerschaftlich sozialisierten Funktionären auf erhebliche Vorbehalte stößt (Greven 2006: 259). Die Bedeutung der internen Opposition steigt dadurch weiter an, als die von den Gewerkschaften inszenierten Kampagnen erfahrungsgemäß erst langfristig Erfolge zeigen. Die beschriebene „Justice for Janitors"-Kampagne lief über einen Zeitraum von zwei Jahren (und läuft in vielen Städten immer noch). Eine derart langfristig angelegte und mit großer Erfolgsunsicherheit behaftete Strategie wird mit starken Veto-Spielern innerhalb der Organisationen schwer zu realisieren sein.

\subsection{Rollenverständnis der Gewerkschaften als intermediäre Organisationen}

Walther Müller-Jentsch hat den Begriff von Gewerkschaften als intermediären Organisationen geprägt (1982). Demnach vermitteln die Arbeitnehmerverbände zwischen Mitgliederinteressen und Systeminteressen; sie sind nicht nur „Gegenmacht“, sondern auch „Ordnungsfaktor“ (Schmidt 1971). Klaus Dörre ist zuzustimmen, dass dies nicht 
mehr das Leitbild der sich revitalisierenden Gewerkschaft ist (2005: 208). ${ }^{3}$ Das korporatistisch geprägte ,,verbandliche Modell sozialer Ordnung“ wird tendenziell ersetzt durch ein Modell sozialer Unordnung, in dem Mitgliederinteressen auch gegen die Kapitalund Systeminteressen durchgesetzt werden sollen. Dies war für die amerikanischen Gewerkschaften nie anders: Weil sie niemals die Chance hatten, politischer Ordnungsfaktor zu sein, blieb ihnen immer nur die Rolle der Gegenmacht. Für die deutschen Gewerkschaften zeichnet sich hier jedoch eine grundlegende Transformation ab, in der die Balance zwischen Systemkompatibilität und Mitgliederinteressen neu zu bestimmen ist, und zwar an beiden Polen des Spannungsfeldes.

Widmen wir uns zuerst der Systemdimension zu: Wie bereits erwähnt wurde, stoßen die Guerilla-Taktiken der amerikanischen Revitalisierungsversuche innerhalb der deutschen Gewerkschaften auf Skepsis. Gerade die Eskalationsstrategie ist häufig jedoch eine zentrale Erfolgsvoraussetzung. Wie berichtet, schlug die öffentliche Stimmung zugunsten der Reinigungskräfte in Los Angeles erst um, als Demonstranten vor laufenden Fernsehkameras von der Polizei misshandelt wurden. Der Zwang zur Eskalation steigt dadurch weiter an, dass die öffentliche Aufmerksamkeit ein flüchtiges Gut ist und viele Organisationen die Kampagnenstrategie für sich entdecken. Bei „Lidl“ musste ver.di erfahren, dass eine schlechte Presse und ein Reputationsschaden keineswegs ausreichten, um das Unternehmen zu einem Strategiewechsel zu bewegen. Hier wäre eine deutlich konfrontativere Strategie nötig gewesen. Ob Gewerkschaften dies wollen sollen und was alternative Druckmittel sein könnten, wenn sie es nicht wollen, ist eine offene Frage.

Doch auch für die Frage der Sozialintegration hat die Transformation Folgen. Die Militanz der SEIU nach außen impliziert eine straff hierarchische Kampagnenorganisation nach innen. Dies verwundert nicht: Zentrale Komponenten der Kampagnenstrategie stammen aus der Praxis erfolgreicher Nichtregierungsorganisationen. Beeinflusst wurde sie zudem von der Managementlehre (Greven 2006: 255). Einige Funktionäre der SEIU wurden sogar an Managementakademien ausgebildet (Waldinger et al. 1998: 109). Weder Unternehmen noch NGOs sind dafür bekannt, demokratisch aufgebaute Organisationen zu sein. Und schon der Begriff „Kampagne“ (französisch für „Feldzug“) legt die Dominanz hierarchischer Planung und Entscheidungsfindung nahe. Zentrale Führungskräfte rekrutieren, mobilisieren und schulen lokale Führungskräfte, um dezentral Führungskompetenzen (nicht aber demokratische Willensbildungs- und Entscheidungsprozesse) auszubauen. Entgegen aller Beteiligungsrhetorik darf kein Zweifel daran bestehen, dass zumindest die ,Janitors“-Kampagne nur wenig mit romantischen Vorstellungen von partizipatorischer Gewerkschaftsdemokratie zu tun hat (Choi 2008: 12). Die strategische Mobilisierung der Basis schließt nicht aus, dass sie eine Eigendynamik entwickelt und die aktivierte Basis auch mehr Demokratie und Mitentscheidungsrechte einfordert. Dennoch ist die Kampagnenpolitik in der amerikanischen Debatte demokratietheoretisch umstritten (vgl. Sharpe 2004: 62 ff.). Auch in Großbritannien, wo Gewerkschaften ebenfalls am US-Modell lernen, wurde kritisiert, bei der Partizipation der Mitglieder handle es sich lediglich um ,managed activism“ (Heery et al. 2000).

3 Allerdings akzentuiert er diese Aussage vor allem normativ. Demnach kann Intermediarität nicht mehr das Leitbild einer sich revitalisierenden Gewerkschaft sein. 
Nun könnte man zu Recht einwerfen, dass Demokratie nie zu den großen gewerkschaftlichen Tugenden zählte. Dies trifft auch und gerade auf die deutschen, einflusslogisch dominierten Gewerkschaften zu. Von daher sind es ja vielleicht gerade die starken Top-down-Elemente, die die amerikanische Revitalisierungsstrategie anschlussfähig an deutsche Traditionen machen. Dabei muss man jedoch bedenken, auf welcher Problemdiagnose die amerikanischen Strategien basieren: Die Gewerkschaften unterstellen, dass sie kein Attraktivitätsproblem bei den Beschäftigten haben, sondern ein Anerkennungsproblem bei den Unternehmen. Diese Vermutung ist nicht unberechtigt. Arbeitnehmerbefragungen zeigen, dass sich die überwältigende Mehrheit der USBeschäftigten eine gewerkschaftliche Interessenvertretung wünscht (Freeman/Rogers 1999). Zudem bekamen im Rahmen der „Janitors“-Kampagne viele Immigranten erstmalig überhaupt einen Zugang zum politischen System. Für diese Beschäftigtengruppe dürfte die Attraktivität der Kampagnenpolitik sehr hoch sein, auch wenn sie zentralistisch gestaltet wird. Und unter der Bedingung, dass viele Unternehmen gewerkschaftsfeindlich gesonnen sind und versuchen, Willensbildungsprozesse mit allen Mitteln zu stören, sind ausgeprägte Bottom-up-Elemente wohl ohnehin (vorerst) zur Erfolglosigkeit verdammt, wie die gewerkschaftlichen Erfahrungen mit den Anerkennungswahlen seit Jahrzehnten zeigen. Vor diesem Hintergrund haben die amerikanischen Gewerkschaften gute Gründe, nach innen und außen als „militante Kampftruppen“ zu agieren. Dies kann bei wohlwollender Betrachtung als Voraussetzung dafür gelten, aus dem „,managed activism“ mittelfristig Gewerkschaftsdemokratie wachsen zu lassen. Ohnehin werden am Ende nur die Gewerkschaften zugelassen, die dazu in der Lage sind, Mehrheiten zu gewinnen (über Anerkennungswahlen oder Card-Check-Verfahren).

Dass diese Diagnose auch den Kern des deutschen Problems trifft, ist jedoch fraglich. Zweifellos ist ein Unternehmen wie der Schwarz-Konzern offen gewerkschaftsfeindlich. Die Gewerkschaften verlieren jedoch bereits seit Mitte der 1980er Jahre Mitglieder. Und Untersuchungen über die individuellen Einstellungen von Beschäftigten gegenüber den Interessenorganisationen zeugen von Distanz. Demnach sehen viele Arbeitnehmer keine Veranlassung, Gewerkschaftsmitglied zu sein, und zwar aus vielen Gründen: weil sie den Flächentarif als Allgemeingut wahrnehmen, weil sie den Betriebsrat als für sie wichtigeren Akteur einschätzen, weil sie eine betriebsnähere Tarifpolitik favorisieren, weil sie die üblichen Verhaltensroutinen bei Tarifverhandlungen ablehnen, weil sie Gewerkschaften als undemokratisch wahrnehmen, weil sie das Verhalten der Gewerkschaften gegenüber den eigenen Mitarbeitern und als Unternehmenseigentümer ablehnen oder weil Skandale die Glaubwürdigkeit der Interessenverbände unterminieren (Holtrup 2005: 21 ff.). Diese Einschätzungen mögen ungerechtfertigt sein oder nicht. Sie legen aber den Schluss nahe, dass deutsche Gewerkschaften nicht nur gegen feindliche Unternehmen antreten, sondern auch um desinteressierte Arbeitnehmer kämpfen. Darum liegt eine beschäftigtenzentrierte Strategie hier näher als eine unternehmenszentrierte Strategie. Wenn eine dreijährige Kampagne lediglich den Erfolg hat, dass einige Hundert (und nicht einige Tausend) Beschäftigte organisiert wurden, stellt sich jedenfalls die Frage, ob die „Lidl“"-Belegschaft überhaupt organisiert werden wollte; und falls nicht, bleibt zu klären, woran das liegt.

Zweifel sind umso mehr angebracht, als innerhalb der DGB-Gewerkschaften mindestens ein alternatives Revitalisierungskonzept praktiziert wird, das in Konkurrenz 
zum importierten US-Modell steht und das den einzelnen Gewerkschaftsmitgliedern weitaus mehr Handlungsspielräume zuweist als nur die Implementation fremdgesteuerter Kampagnen. Die IG Metall versucht in einigen Bezirken, die Input-Legitimität zu stärken und ihre organisatorische Basis durch eine Bottom-up-Strategie zu stabilisieren. Sie lässt ihre Mitglieder im Rahmen einer betrieblichen Tarifpolitik bei Bedarf über Tarifverträge abstimmen und die betriebliche Tarifpolitik selbst gestalten. Das Konzept der betrieblichen Tarifpolitik basiert auf der Annahme, dass die Gewerkschaftsmitglieder in den Betrieben (und kein stellvertretend agierender Funktionär) für die Qualität ihrer Tarifverträge verantwortlich sind. So wie in den USA wird dabei auch hier mit dem Partizipationsansatz aus der Not eine Tugend gemacht. Betriebliche Tarifpolitik findet dort statt, wo zentral ausgehandelte Branchentarifverträge an Prägekraft verloren haben. Da die gewerkschaftlichen Funktionärskapazitäten nicht ausreichen, um die Dezentralisierung des Tarifsystems zentral zu steuern, ergibt sich die Notwendigkeit, dass die Gewerkschaftsmitglieder vor Ort ihre eigene Tarifpolitik bestimmen. Damit wird gleichzeitig die Hoffnung verknüpft, dass die Gewerkschaft als Organisation an Attraktivität gewinnt, wenn die Mitglieder ihre Arbeitsbedingungen selbst gestalten können.

Man sollte die Differenzen zwischen ver.di und der IG Metall nicht überbewerten. Auch die IG Metall zeigt sich interessiert an Kampagnenpolitik, und bei ver.di existiert heute mehr Beteiligung als früher. Der grundlegende Unterschied zwischen beiden Organisationen und zu den USA besteht aber darin, dass die IG Metall die Beschäftigten ins Zentrum stellt (nicht Unternehmen), die Partizipation der Basis ergebnisoffen ist und Entscheidungsrechte beinhaltet (während ver.di vor allem die gesteuerte Mobilisierung anstrebt) und die IG Metall-Beschäftigten in erheblichem Maße ihre eigenen Entscheidungen treffen, also in das gewerkschaftliche Kerngeschäft eingreifen.

\subsection{Grundlagen der Interessenorganisation}

Die US-Kampagnen basieren auf der Leitidee, die attackierten Unternehmen als lokal eingebettete Organisationen zu betrachten. Zwar ist die „Justice for Janitors“-Initiative eine nationale Kampagne. Ihre Erfolgsfaktoren liegen jedoch stark in lokalen Kontexten begründet: Lokale Arbeitsmärkte zu kontrollieren, gewerkschaftsfeindliche (aber lokal gebundene) Arbeitgeber in ihrem Nahbereich (Restaurants, Sportclubs etc.) zu diskreditieren, die Aufmerksamkeit der lokalen Medien auf sich zu ziehen und lokal politische Krisen zu inszenieren sind Strategien, die auf eine Strukturgrundlage der Interessenpolitik hinweisen, die auch für das amerikanische berufs- und betriebszentrierte System eine neue Qualität hat. Mit dem Revitalisierungskonzept nähern sich die US-Gewerkschaften einem distriktbezogenen Modell der industriellen Beziehungen an, wie es von Wolfgang Streeck als vierter Typus interessenpolitischer Organisation beschrieben wurde (1993: 50 ff.). Gemeinsame Interessen und Identitäten der Arbeitnehmer werden dabei nicht mehr entlang der Klasse, des Berufes oder des Betriebs definiert, sondern auf der Basis der Region - d. h. identitätsstiftend sind örtliche Nähe und kulturelle Vertrautheit. Es ist zwar erstaunlich, doch trotzdem kein Zufall, dass in den USA vor allem lateinamerikanische Immigranten organisiert werden konnten, die durch sozialstrukturelle und familiäre Netzwerke sozial integriert sind. 
Die Erschließung lokaler Ressourcen wird durch die starke Fragmentierung des politischen Systems und der Medienlandschaft begünstigt. Krisen zu initiieren und sie mediengerecht in Szene zu setzen, ist in den USA ungleich leichter als in Zentralstaaten, weil Tageszeitungen kaum über den regionalen Nahbereich hinausreichen. Eine lokal ausgerichtete Strategie wäre auch in Deutschland vorstellbar, da sich die Regionen trotz Bundesstaatsprinzip immer starke Eigenheiten bewahrt haben. In verschiedenen Regionen hätte eine Bewegungsgewerkschaft gute Aussichten, erfolgreich zu mobilisieren, auch wenn eine - mit den USA vergleichbare - sozialstrukturelle Basis fehlt. Man denke z. B. an Bochum, wo anlässlich der Konflikte bei Nokia und Opel die ganze Stadt (inklusive des ortsansässigen Fußballvereins) auf den Beinen war, um die protestierenden Arbeitnehmer zu unterstützen. Im Hamburger Stadtteil St. Pauli ist es einer Koalition aus Anwohnern, Gewerkschaftern und anderen sozial(politisch)en Gruppen gelungen, die Schließung eines Krankenhauses zu verhindern. Aus dieser Initiative erwuchs eine breitere Protestbewegung gegen die Arbeitsbedingungen der Beschäftigten in den privatisierten Hospitälern (Greer 2008). Ähnliches wäre denkbar in Berlin-Kreuzberg oder in der Kölner Südstadt.

Auch die „Lidl“-Kampagne arbeitet nicht ohne einen lokalen Bezug. Es wurde nicht darauf abgestellt, alle Betriebe Deutschlands zu organisieren. Vielmehr wurden lokale Aktivitätszentren identifiziert, auf die sich die Bemühungen konzentrierten. Trotzdem wurde die stärkste Ressource amerikanischer Kampagnen, der Distrikt, nicht ausgebeutet, weil davon abgesehen wurde, die Auseinandersetzung in lokale Konflikte einzubetten und in diesem Zusammenhang lokale Entscheidungsträger in den Konflikt hineinzuzwingen. Dafür hat die Gewerkschaft auch gute Gründe. Für die amerikanischen berufs- und betriebszentrierten Gewerkschaften ist jede regionale Interessenorganisation, die dabei hilft, lokale Arbeitsmärkte vor ruinöser Konkurrenz zu schützen, ein Zentralisierungsgewinn. Für eine deutsche klassenbasierte Einheitsgewerkschaft, die in der Vergangenheit bereits eine betriebsbezogene Dezentralisierung hinnehmen musste, bedeutet das Distriktmodell der Arbeitsbeziehungen hingegen weitere Zentralisierungsverluste. Gleiche Arbeitsbedingungen würden in diesem Modell nicht nur nicht mehr für alle Beschäftigten der Branche gelten, sondern auch nicht mehr für alle Beschäftigten eines Unternehmens. Die Beschäftigten der „Lidl“-Supermärkte Kölns würden eventuell unter völlig anderen Bedingungen arbeiten als die „Lidl“-Beschäftigten in Berlin. Zusammengefasst: Wenn deutsche Gewerkschaften die zentralen Machtressourcen amerikanischer Bewegungsgewerkschaften für sich nutzen wollen, wirft dies normativ-strategische Fragen in Bezug auf die zukünftige Identität der deutschen Gewerkschaften auf, die nicht einfach zu beantworten sein werden.

\section{Schluss}

Das Bild von den Gewerkschaften als „Dinosaurier der Dienstleistungsgesellschaft“ ist korrekturbedürftig. In vielen Ländern arbeiten sie daran, aus organisationalen Routinen auszubrechen und sich gerade im Dienstleistungssektor neu zu etablieren. Gewerkschaften sind damit heute besonders interessante Beispiele für Prozesse des kulturübergrei- 
fenden Organisationslernens. Der Aufsatz beschäftigte sich mit einem Aspekt dieser Entwicklung, dem Versuch der Dienstleistungsgewerkschaft ver.di, von amerikanischen Schwesterorganisationen zu lernen, Mitgliederzuwächse mittels strategischer Kampagnenpolitik im Rahmen eines breit angelegten Revitalisierungskonzepts zu erzielen.

Kann die US-Strategie auch im deutschen Kontext funktionieren? Der Aufsatz argumentiert, dass der Erfolg amerikanischer Gewerkschaften auf einigen zentralen Voraussetzungen basiert, die in Deutschland nicht gegeben oder prekär sind: Erstens sind hierarchisch gesteuerte Prozesse des Organisationslernens in deutschen Gewerkschaften schwieriger durchsetzbar als in den USA. Zweitens basieren die US-Kampagnen auf der Problemdiagnose, dass die gewerkschaftsfeindliche Einstellung vieler Unternehmen die zentrale Organisationsrestriktion darstellt, die es zu überwinden gilt. Deutsche Gewerkschaften haben es hingegen nicht nur (und wahrscheinlich nicht einmal in erster Linie) mit ablehnenden Arbeitgebern zu tun, sondern auch mit desinteressierten Arbeitnehmern. Dies legt die Strategie nahe, nicht Unternehmen, sondern Beschäftigte ins Zentrum einer Kampagne zu stellen. Und drittens ging der Strategiewechsel US-amerikanischer Gewerkschaftspolitik mit einer schleichenden Transformation der Grundlagen interessenpolitischer Organisation einher. Die Region bildet den Fokus der Arbeitnehmeridentität und die Machtressource der Kampagnenpolitik. Dieser Weg stünde auch den deutschen Gewerkschaften prinzipiell offen. Sie würden dafür aber einen deutlich höheren Preis zahlen müssen als die amerikanischen Verbände.

Gerade im Dienstleistungsbereich erleben wir gegenwärtig, dass die Berufsgewerkschaften organisationsstärker werden und dass auch solche Beschäftigtengruppen für ihre Arbeitnehmerrechte eintreten, von denen man dies nicht unbedingt erwartet hätte (vor allem die Ärzte). Diese Entwicklung zeigt, dass es ein nicht voll ausgeschöpftes Organisationspotenzial gibt. Und eine Einheitsgewerkschaft müsste sich die Frage stellen, warum es ihr nicht (mehr) gelingt, diese Gruppen anzusprechen und zu repräsentieren. Offen ist, ob eine Kampagnenpolitik, die vor allem nach außen gerichtet ist, diese nach innen gerichtete Frage überhaupt in den Blick nimmt.

\section{Literatur}

Argyris, Chris/Donald A. Schön (1999): Die lernende Organisation. Grundlagen, Methode. Praxis. Stuttgart: Klett-Cotta.

Artus, Ingrid (2001): Krise des deutschen Tarifsystems. Die Erosion des Flächentarifvertrags in Ost und West. Wiesbaden: Westdeutscher Verlag.

Bandelow, Nils (2003): Politisches Lernen und politische Veränderungen. In: Klaus Schubert/ Nils Bandelow (Hrsg.), Lehrbuch der Politikfeldanalyse. München u.a.: Oldenbourg, S. 289-331.

Beckert, Jens (1999): Agency, Entrepreneurs, and Institutional Change. The Role of Strategic Choice and Institutionalized Practices in Organizations. In: Organization Studies 20, S. 777-799.

Bsirske, Frank (2005): Grußwort an die Teilnehmer und Teilnehmerinnen der Tagung „Kampagnen - eine Kampfform der Gewerkschaften und sozialen Bewegungen“. Oberjosbach. November 2005. 
Bremme, Peter (2007): Respekt und bessere Jobs. Organizing im Hamburger Sicherheitsgewerbe. In: Peter Bremme/Ulrike Fürniß/Ulrich Meinecke (Hrsg.), Never Work Alone. Organizing - ein Zukunftsmodell für Gewerkschaften. Hamburg: VSA Verlag, S. 194-217.

Bremme, Peter/Ulrike Fürniß/Ulrich Meinecke (Hrsg.) (2007a): Never Work Alone. Organizing - ein Zukunftsmodell für Gewerkschaften. Hamburg: VSA Verlag.

Bremme, Peter/Ulrike Fürniß/Ulrich Meinecke (2007b): Organizing als Zukunftsprogramm für bundesdeutsche Gewerkschaften. In: dies. (Hrsg.), Never Work Alone. Organizing - ein Zukunftsmodell für Gewerkschaften. Hamburg: VSA Verlag, S. 10-23.

Bronfenbrenner, Kate/Sheldon Friedman/Richard W. Hurd/Rudolph A. Oswald/Ronald L. Seeber (Hrsg.) (1998): Organizing to Win. New Research on Union Strategies. Ithaca, NY u.a.: ILR Press.

Bronfenbrenner, Kate/Robert Hickey (2004): Changing to Organize. A National Assessment of Union Strategies. In: Ruth Milkman/Kim Voss (Hrsg.), Rebuilding Labor. Organizing and Organizers in the New Union Movement. Ithaca, NY u.a.: Cornell University Press, S. 17-61.

Choi, Hae-Lin (2008): Re-thinking Justice for Janitors - Strategische Erneuerung der amerikanischen Dienstleistungsgewerkschaft SEIU. In: WSI-Mitteilungen 61, S. 11-17.

DiMaggio, Paul (1988): Interest and Agency in Institutional Theory. In: Lynne Zucker (Hrsg.), Institutional Patterns and Organizations. Cambridge, Mass: Ballinger, S. 3-32.

Dörre, Klaus (2005): Intermediarität und gewerkschaftliche Identität. In: Industrielle Beziehungen 12, S. 207-212.

Dörre, Klaus (2006): Revitalisierung von Gewerkschaften. Aus der Krise zur Erneuerung? Thesenpapier zur Tagung „Revitalisierung von Gewerkschaften“. Jena, Dezember 2006 (unveröffentlicht).

Dribbusch, Heiner (2007): Das „Organizing-Modell““. Entwicklung, Varianten und Umsetzung. In: Peter Bremme/Ulrike Fürniß/Ulrich Meinecke (Hrsg.), Never Work Alone. Organizing - ein Zukunftsmodell für Gewerkschaften. Hamburg: VSA Verlag, S. 24-52.

Ebbinghaus, Bernhard (2002): Dinosaurier der Dienstleistungsgesellschaft. Der Mitgliederschwund deutscher Gewerkschaften im historischen und internationalen Vergleich. In: MPIfG Working Paper 02/3.

Ebbinghaus, Bernhard/Jelle Visser (1999): When Institutions Matter. Union Growth and Decline in Western Europe, 1950-1995. In: European Sociological Review 15, S. 135-158.

Ellguth, Peter/Kohaut, Susanne (2007): Tarifbindung und betriebliche Interessenvertretung. Aktuelle Ergebnisse aus dem IAB-Betriebspanel 2006. In: WSI-Mitteilungen 60, S. 511-514.

Erickson, Christopher L./Catherine L. Fisk/Ruth Milkman/Daniel J.B. Mitchell/Kent Wong (2008): Unions and Low-wage Immigrant Workers: Lessons from the Justice for Janitors Campaign in Los Angeles, 1990-2002, revised 2008. http://www.law.berkeley.edu/institutes/ csls/Fisk\%20paper.doc, Juni 2008.

Fletcher, Bill Jr./Richard W. Hurd (1998): Beyond the Organizing Model. The Transformation Process in Local Unions. In: Kate Bronfenbrenner/Sheldon Friedman/Richard W. Hurd/Rudolph A. Oswald/Ronald L. Seeber (Hrsg.), Organizing to Win. New Research on Union Strategies. Ithaca, NY u.a.: ILR Press, S. 37-53.

Freeman, Richard B./Joel Rogers (1999): What Workers Want. Ithaca, NY u.a.: ILR Press.

Frege, Carola M. (2000): Gewerkschaftsreformen in den USA. Eine kritische Analyse des „Organisationsmodells“. In: Industrielle Beziehungen 7, S. 260-280.

Frege, Carola M./John Kelly (Hrsg.) (2003): Union Revitalization Strategies in Comparative Perspective. Special Issue of European Journal of Industrial Relations 9. London: Sage.

Goerke, Laszlo/Markus Pannenberg (2007): Trade Union Membership and Works Councils in West Germany. In: Industrielle Beziehungen 14, S. 154-175.

Greer, Ian (2008): Von sozialen Bewegungen lernen: Ein Impuls für deutsche Gewerkschaften. In: WSI-Mitteilungen 61, S. 205-211. 
Greven, Thomas (2006): U.S. Unions' Strategic Campaigns against Transnational Enterprises in Germany. In: Industrielle Beziehungen 13, S. 253-269.

Hälker, Juri/Claudius Vellay (Hrsg.) (2006): Union Renewal - Gewerkschaften in Veränderung. Texte aus der aktuellen internationalen Gewerkschaftsforschung. Düsseldorf: Hans-Böckler-Stiftung.

Hall, Peter/David Soskice (Hrsg.) (2001a): Varieties of Capitalism. The Institutional Foundations of Comparative Advantage. Oxford: Oxford University Press.

Hall, Peter/David Soskice (2001b): An Introduction to Varieties of Capitalism. In: dies. (Hrsg.), Varieties of Capitalism. The Institutional Foundations of Comparative Advantage. Oxford: Oxford University Press, S. 1-70.

Heery, Edmund/Melanie Simms/Rick Delbridge/John Salmon/Dave Simpson (2000): Union Organizing in Britain. A Survey of Theory and Practice. In: International Journal of Human Resource Management 11, S. 986-1007.

Holtrup, André (2005): Interessen und Interessenvertretung heute - aus der Perspektive von Beschäftigten. Erste empirische Befunde aus dem Forschungsprojekt zur subjektiven Relevanz von Arbeitsregulierung. IAW Arbeitspapier 16, Universität Bremen.

Katz, Harry C./Thomas A. Kochan (2000): An Introduction to Collective Bargaining and Industrial Relations. Boston, Mass. u.a.: IRWIN/Mc Graw-Hill.

Levitt, Martin J.(1993): Confessions of a Union Buster. New York: Crown.

Martin, Andrew/George Ross (Hrsg.) (1999): The Brave New World of European Labor. European Trade Unions at the Millenium. New York/Oxford: Berghahn Books.

Milkman, Ruth (2006): Resurgent Unionism in an Immigrant City. L.A. Exceptionalism and the Future of the U.S. Labor Movement. Paper for the Conference of the Council for European Studies. Chicago, March 2006.

Milkman, Ruth/Kim Voss (2004): Introduction. In: Ruth Milkman/Kim Voss (Hrsg.), Rebuilding Labor. Organizing and Organizers in the New Union Movement. Ithaca, NY u.a.: Cornell University Press, S. 1-16.

Milkman, Ruth/Kent Wong (2001): Organizing Immigrant Workers. Case Studies from Southern California. In: Lowell Turner/Harry C. Katz/Richard W. Hurd (Hrsg.), Rekindling the Movement. Labor's Quest for Relevance in the 21st Century. Ithaca, NY u.a.: ILR Press, S. 99-128.

Müller-Jentsch, Walther (1982): Gewerkschaften als intermediäre Organisationen. In: Gert Schmidt/Hans-Joachim Braczyk/Jost von dem Knesebeck (Hrsg.), Materialien zur Industriesoziologie. Kölner Zeitschrift für Soziologie und Sozialpsychologie, Sonderheft 24, S. 408-432.

Nissen, Bruce (1998): Utilizing the Membership to Organize the Unorganized. In: Kate Bronfenbrenner/Sheldon Friedman/Richard W. Hurd/Rudolph A. Oswald/Ronald L. Seeber (Hrsg.), Organizing to Win. New Research on Union Strategies. Ithaca, NY u.a.: ILR Press, S. $135-149$.

Olsen, Mancur (1968): The Logic of Collective Action. Public Goods and the Theory of Groups. New York: Schocken Books.

Oxenbridge, Sarah (1997): Organising Strategies and Organising Reform in New Zealand Service Sector Unions. In: Labor Studies Journal 22, S. 3-27.

Raffo, Jeffrey (2007): Organizingerfahrungen aus Nordamerika - und aus Nordrhein-Westfalen. In: Peter Bremme/Ulrike Fürniß/Ulrich Meinecke (Hrsg.), Never Work Alone. Organizing - ein Zukunftsmodell für Gewerkschaften. Hamburg: VSA Verlag, S. 175-193.

Raschke, Joachim (1985): Soziale Bewegungen. Ein historisch-systematischer Grundriss. Frankfurt a.M./New York: Campus.

Regini, Marino (Hrsg.) (1992): The Future of Labour Movements. London: Sage. 
Rehder, Britta (2006): Legitimitätsdefizite des Co-Managements. Betriebliche Bündnisse für Arbeit als Konfliktfeld zwischen Arbeitnehmern und betrieblicher Interessenvertretung. In: Zeitschrift für Soziologie 35, S. 227-242.

Rickens, Christian (2006): Verdi und die Veganer. In: managermagazin 3/2006, S. 126-133.

Rudy, Preston (2004): „Justice for Janitors“, Not „Compensation for Custodians“. The Political Context and Organizing in San José and Sacramento. In: Ruth Milkman/Kim Voss (Hrsg.), Rebuilding Labor. Organizing and Organizers in the New Union Movement. Ithaca, NY u.a.: Cornell University Press, S. 133-149.

Scharpf, Fritz W. (1970): Demokratietheorie zwischen Utopie und Anpassung. Konstanz: Universitätsverlag.

Schmidt, Eberhard (1971): Ordnungsfaktor oder Gegenmacht. Die politische Rolle der Gewerkschaften. Frankfurt a.M.: Suhrkamp.

Schmitter, Philippe/Wolfgang Streeck (1999): Gemeinschaft, Markt, Staat - und Verbände? Der mögliche Beitrag von privaten Interessenregierungen zu sozialer Ordnung. In: Wolfgang Streeck (Hrsg.), Korporatismus in Deutschland. Zwischen Nationalstaat und Europäischer Union. Frankfurt a.M./New York: Campus, S. 191-222.

Schnabel, Claus/Joachim Wagner (2007): The Persistent Decline in Unionization in Western and Eastern Germany, 1980-2004: What Can We Learn from a Decomposition Analysis? In: Industrielle Beziehungen 14, S. 118-132.

Schreieder, Agnes (2005): Organizing - Gewerkschaft als soziale Bewegung. Berlin: ver.di Bundesverwaltung.

Schreieder, Agnes (2007): Die Lidl-Kampagne - ein Zukunftsmodell für Gewerkschaften. In: Peter Bremme/Ulrike Fürniß/Ulrich Meinecke (Hrsg.), Never Work Alone. Organizing - ein Zukunftsmodell für Gewerkschaften. Hamburg: VSA Verlag, S. 153-174.

Sharpe, Theresa (2004): Union Democracy and Successful Campaigns. The Dynamics of Staff Authority and Worker Participation in an Organizing Union. In: Ruth Milkman/Kim Voss (Hrsg.), Rebuilding Labor. Organizing and Organizers in the New Union Movement. Ithaca, NY u.a.: Cornell University Press, S. 62-87.

Stövesand, Sabine (2007): (Für den) Blick über den Tellerrand. Organizing im lokalen Gemeinwesen. In: Peter Bremme/Ulrike Fürniß/Ulrich Meinecke (Hrsg.), Never Work Alone. Organizing - ein Zukunftsmodell für Gewerkschaften. Hamburg: VSA Verlag, S. 79-91.

Streeck, Wolfgang (1993): Klasse, Beruf, Unternehmen, Distrikt. Organisationsgrundlagen industrieller Beziehungen im europäischen Markt. In: Burkhard Stümpel/Meinolf Dierkes (Hrsg.), Innovation und Beharrung in der Arbeitsmarktpolitik. Stuttgart: Schäffer Poeschel, S. $39-68$.

Turner, Lowell/Richard W. Hurd (2001): Building Social Movement Unionism. The Transformation of the American Labor Movement. In: LowellTurner/Harry C. Katz/Richard W. Hurd (Hrsg.), Rekindling the Movement. Labor's Quest for Relevance in the 21st Century. Ithaca, NY u.a.: ILR Press, S. 9-26.

Ver.di, Vereinte Dienstleistungsgewerkschaft (2005): Konsequenzen der Organising-Woche. Aus dem Beschluss des Landesbezirksvorstandes Hamburg. o.D. Hamburg.

Visser, Jelle (1995): Trade Unions from a Comparative Perspective. In: Joris van Ruysseveldt/Rien Huiskamp/Jacques van Hoof (Hrsg.), Comparative Industrial and Employment Relations. London: Sage, S. 37-67.

Voss, Kim/Rachel Sherman (2000): Breaking the Iron Law of Oligarchy: Union Revitalization in the American Labor Movement. In: American Journal of Sociology 106, S. 303-349.

Waldinger, Roger/Chris Erickson/Ruth Milkman/Daniel J.B. Mitchell/Abel Valenzuela/Kent Wong/Maurice Zeitlin (1998): Helots no More. A Case Study of the Justice for Janitors Campaign in Los Angeles. In: Kate Bronfenbrenner/Sheldon Friedman/Richard W. Hurd/Rudolph A. Oswald/Ronald L. Seeber (Hrsg.), Organizing to Win. New Research on Union Strategies. Ithaca, NY u.a.: ILR Press, S. 102-119. 
Woodruff, Tom (2007): Gewerkschaftsaufbau in schwierigen Zeiten. Wie die SEIU zur Gewerkschaft mit den höchsten Mitgliederzuwächsen in den USA wurde. In: Peter Bremme/Ulrike Fürniß/Ulrich Meinecke (Hrsg.), Never Work Alone. Organizing - ein Zukunftsmodell für Gewerkschaften. Hamburg: VSA Verlag, S. 92-116.

\section{Britta Rehder}

Geb. 1970. Wissenschaftliche Mitarbeiterin am Max-Planck-Institut für Gesellschaftsforschung Köln. Forschungsschwerpunkte: Verbände, Arbeitsbeziehungen, Politik und Recht. Ausgewählte Veröffentlichungen: Legitimitätsdefizite des Co-Managements. Betriebliche Bündnisse für Arbeit als Konfliktfeld zwischen Arbeitnehmern und betrieblicher Interessenvertretung. In: Zeitschrift für Soziologie 35, 2006; Recht und Politik beim Wandel des Flächentarifs. Juristen als politische Akteure im System der Arbeitsbeziehungen. In: Politische Vierteljahresschrift 47, 2006; What is Political about Jurisprudence? Courts, Politics, and Political Science in Europe and the United States. MPIfG Discussion Paper 07/5. 